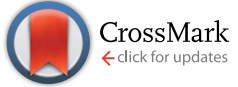

Cite this: RSC Adv., 2016, 6, 104164

Received 26th August 2016 Accepted 22nd October 2016

DOI: $10.1039 / c 6 r a 21427 d$

www.rsc.org/advances

\section{Dye-doped silica nanoparticle probes for fluorescence lifetime imaging of reductive environments in living cells $\uparrow$}

\author{
Luca Petrizza, ${ }^{\text {ab }}$ Mayeul Collot, ${ }^{* a}$ Ludovic Richert, $^{a}$ Yves Mely, ${ }^{a}$ Luca Prodib \\ and Andrey S. Klymchenko*a
}

Fluorescence detection sensitivity can be drastically improved by the application of nanoparticles (NPs) because of their superior brightness compared to organic dyes. Here, using dye-doped silica NPs (SiNPs), we developed FRET-based nanoparticle probes for the detection of reductive environments in living cells. To this end, we designed three FRET acceptors based on black hole quenchers (BHQs). Their polarity was tuned by introducing hydroxyl, PEG and sulfate groups. To conjugate them to NPs, we used an original pre-functionalization approach, where the quencher was coupled by a "click" reaction to Pluronic F127 and further used for the preparation of silica NPs. This approach enabled easy preparation of silica NPs functionalized with varying amounts of quenchers by simple mixing of functionalized and parent Pluronic F127 in different mol\%. The increase in the quencher concentration at the SiNPs surface produced a rapid drop in the fluorescence intensity with $80 \%$ quenching and a 2 -fold drop in the emission lifetime for $16 \mathrm{~mol} \%$ of the quenchers. Then, to obtain turn-ON sensing of reductive environments, the quenchers were coupled to the NPs through a disulfide linker using the same prefunctionalization strategy. The obtained nano-probes showed a $>10$-fold increase in their fluorescence in the presence of reductive agents, such as tris(2-carboxyethyl)phosphine (TCEP) and glutathione. Remarkably, BHQ quencher bearing sulfate group showed the highest turn-ON response, probably due to its superior capacity to escape from the NP surface after disulfide bond cleavage. The obtained best nanoprobe was successfully applied for detection of reductive environments inside living cells using fluorescence lifetime imaging (FLIM). This work provides insights for FRET acceptor design and its controlled grafting, which enables preparation of the first redox-sensitive silica nanoparticle probe for lifetime imaging.

\section{Introduction}

Fluorescent probes are essential tools in current biomolecular research. ${ }^{1-3}$ They enable detection of bioactive molecules and monitoring of biomolecular processes with the highest specificity and sensitivity. However, the ever present problem of fluorescent molecular probes is their brightness, due to the limited values of their extinction coefficient and quantum yields. ${ }^{4}$ Therefore, fluorescent nanoparticles have gained recent attention because they can be 10-100 fold brighter than individual dyes. $^{5-7}$ So far, a variety of fluorescent NPs has been developed: quantum dots (QDs), ${ }^{\mathbf{8} 9}$ dye-doped silica NPs

${ }^{a}$ Laboratoire de Biophotonique et Pharmacologie, UMR 7213 CNRS, Université de Strasbourg, Faculté de Pharmacie, 74, Route du Rhin, 67401 Illkirch Cedex, France. E-mail: andrey.klymchenko@unistra.fr; mayeul.collot@unistra.fr

${ }^{b}$ Dipartimento di Chimica “Giacomo Ciamician”, Università degli Studi di Bologna, via Selmi 2, 40126 Bologna, Italy

† Electronic supplementary information (ESI) available. See DOI: $10.1039 / \mathrm{c} 6 \mathrm{ra} 21427 \mathrm{~d}$
(SiNPs),${ }^{10-13}$ nanodiamonds, ${ }^{14}$ conjugated polymer NPs,${ }^{15,16}$ dyedoped polymer NPs, ${ }^{7,17-19}$ etc. Within this list, dye-loaded silica NPs present particular interest due to the high brightness and photostability they can reach, combined with the very low toxicity of many of their formulations. Moreover, their high versatility offered by the possible synthetic strategies and by the introduction of effective collective photophysical processes ${ }^{\mathbf{1 8 , 2 0}}$ makes them a powerful toolbox for addressing many different imaging and sensing applications in the field of nanomedicine.

The most common mechanism in the design of fluorescent probes is Förster Resonance Energy Transfer FRET. ${ }^{21}$ It is particularly suitable for molecular probes because dyes are very small entities, which enable preparation of FRET pairs with sufficiently small inter-fluorophore distances that ensure efficient FRET. ${ }^{22-24}$ In contrast, NPs are less efficient FRET donors or acceptors because frequently their size is much larger than the Förster radius. Thus, earlier works on quantum dots showed that efficient FRET requires $\geq 10$ FRET acceptors at the periphery of the organic shell, ${ }^{25,26}$ though there are examples for high FRET with fewer acceptors, but located just next to the 
inorganic core of NPs. ${ }^{27}$ Dye-doped silica NPs are particularly promising FRET donors for two reasons. At first, recent protocols that use organic surfactant Pluronic as template enable preparation of ultrasmall NPs down to $10 \mathrm{~nm}$ size. ${ }^{11}$ Second, at high dye loading energy hopping within the encapsulated dyes can further improve the efficiency of the energy transfer. ${ }^{20,28}$ As we have demonstrated, this process, due to the energy transfer among chromophores of the same nature (the so-called "homoFRET") plays in fact a prominent role in distributing the excitation energy among several donor dyes, including the closest ones to the final acceptors. ${ }^{20}$ In this way, the silica NPs can be employed as "excitation energy reservoirs", leading to nanoarchitectures that can be advantageously designed, for example, as nanosensors for ions, ${ }^{29,30} \mathrm{pH}^{31}$ small bioactive molecules, ${ }^{32,33}$ nucleic acids, ${ }^{34}$ etc. as well as photoswitchable systems, ${ }^{35}$ large Stokes shift emitters, ${ }^{36,37}$ and fluorescent drug delivery systems. ${ }^{38-40}$

Herein we describe the development of FRET-based nanosensors using rhodamine-doped silica nanoparticles (SiNPs) linked to amphiphilic BHQ-2 type quenchers through a reducible disulfide (S-S) group. Disulfide bond has been proven to be a robust cleavable bond in the reductive environment of the cells. ${ }^{41-43}$ We varied the polarity of the quenchers by adding different polar heads and studied their interaction with fluorescent SiNPs as well as their ability to quench their fluorescence by direct adsorption and by covalent linkage. These nanosensors displayed a fluorescence increase (turn-ON effect) upon reduction of the disulfide bond by tris(2-carboxyethyl) phosphine (TCEP) or glutathione that separate the BHQ quencher from the particles. Moreover, this quenching system showed significant effect on the fluorescence lifetime of the SiNPs, which allowed us to use this system in Fluorescence Lifetime Imaging Microscopy (FLIM) experiments in live cells.

\section{Results and discussion}

\section{Design of the nanoprobes}

We designed a system where a fluorescent SiNP bears covalently linked quenchers through a disulfide bond. Upon reduction of this bond, the quenchers separate from the particle and provoke a fluorescence increase as the particle recovers its initial fluorescence. As a particle we used previously reported rhodamine B-doped SiNPs, prepared using Pluronic F127 as a scaffold..$^{36}$ These NPs feature $\sim 10 \mathrm{~nm}$ core diameter and $\sim 5 \mathrm{~nm}$ size PEG shell. In order to achieve an efficient turn-ON of these SiNPs, the quencher must fulfil several properties. First, the overlap between the particle's emission spectrum and the absorbance spectrum of the quencher must be sufficient to insure an appropriate quenching effect. Therefore, Black Hole Quencher-2 (BHQ-2) was chosen as FRET acceptor. Then, the quencher should present an intermediate polarity, so that it could locate inside the PEG shell and thus close to the fluorescent core of the SiNPs to ensure efficient FRET. On another hand, once the disulfide bond get cleaved, the quencher moiety must be sufficiently hydrophilic so that it can escape from the particle in order to recover the fluorescence of SiNPs providing an optimal turn-ON effect. However, BHQ quenchers are noncharged apolar molecules and therefore have a lipophilic nature that could be detrimental for their escape from SiNPs. We consequently designed and synthesised three different functionalizable and amphiphilic quenchers: $\mathrm{BHQ}-\mathrm{OH}, \mathrm{BHQ}-$ $\mathrm{SO}_{3}$ and $\mathrm{BHQ}-\mathrm{PEG}$ that bear, in addition to a "clickable" alkyne group, different polar heads, i.e., hydroxyl, sulphate and methoxy- $\mathrm{PEG}_{8}$ groups, respectively (Fig. 1).

At the first step of the synthesis of BHQ-OH, N-(2-hydroxyethyl)aniline was reacted with propargyl bromide to introduce a clickable group. The obtained aniline was reacted with Fast Black K giving rise to $\mathrm{BHQ}-\mathrm{OH}$ (Scheme 1). BHQ-OH was efficiently converted to BHQ- $\mathrm{SO}_{3}$ by sulfation using the $\mathrm{SO}_{3} \cdot \mathrm{NEt}_{3}$ complex with a yield of $87 \%$. BHQ-OH was clicked to a methoxy $\mathrm{PEG}_{8}$ moiety to afford compound 3 . The latter was then converted into an active carbonate 4 , which was coupled to propargylamine to obtain BHQ-PEG (Scheme 1).

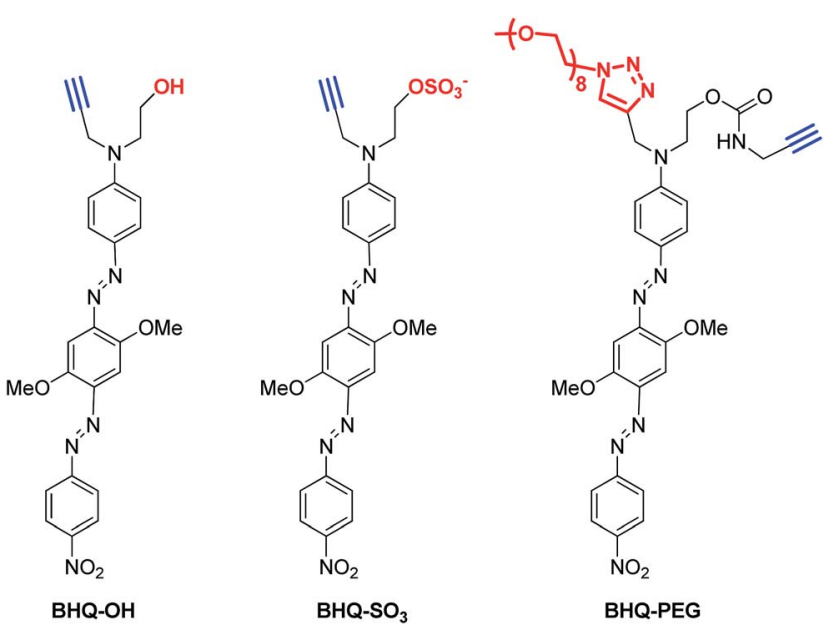

Fig. 1 Structure of the 3 amphiphilic functionalizable BHQs. Polar heads are highlighted in red, the functionalizable site is highlighted in blue.

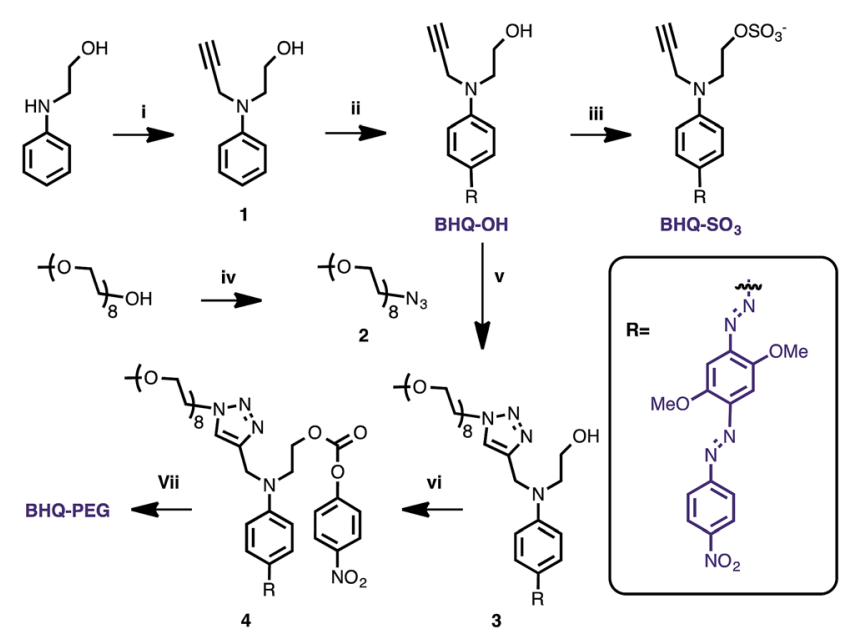

Scheme 1 Synthesis of the 3 "clickable" BHQs: (i) propargyl bromide, $\mathrm{NaHCO}_{3}, \mathrm{DMSO}$; (ii) Fast Black $\mathrm{K}$ salt, dioxane/water; (iii) $\mathrm{SO}_{3} \cdot \mathrm{NEt}_{3}$, DMF; (iv) $\mathrm{SOCl}_{2}$, pyridine, $\mathrm{CHCl}_{3}, \mathrm{NaN}_{3}$, DMF (2 steps); (v) 2, $\mathrm{CuSO}_{4}$ - $5 \mathrm{H}_{2} \mathrm{O}$, Asc Na, dioxane/water; (vi) 4-nitrophenyl chloroformate, pyridine, DCM; (vii) propargylamine, DIEA, DMF. 


\section{Model FRET experiments}

These amphiphilic quenchers were first used to study their ability to interact non-specifically with the surface of SiNPs. Increasing amounts of BHQs were added to an aqueous solution of rhodamine B doped SiNPs and the fluorescence spectra were recorded. It was found that the quenching efficiency was dependent on the structure of the quencher (Fig. 2). At a ratio of two BHQ molecules per SiNP (10 rhodamine B dyes per SiNP,

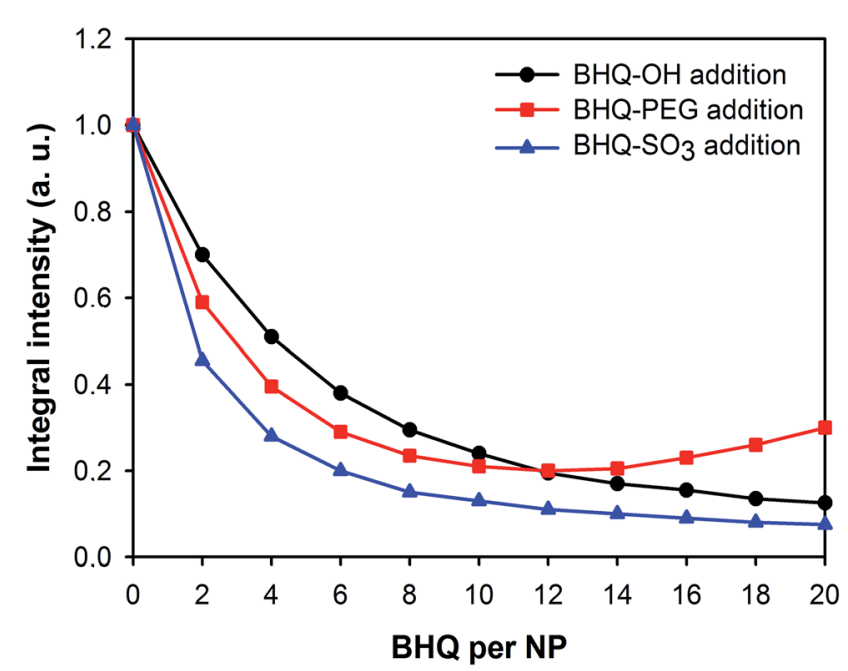

Fig. 2 Effect of the adsorption of the $3 \mathrm{BHQs}$ on the SiNPs' fluorescence intensity (excitation wavelength $=530 \mathrm{~nm}$ ); concentration of $\mathrm{SiNPs}=200 \mathrm{nM}$. calculated as previously reported ${ }^{36}$ ), BHQ-OH, BHQ-PEG and BHQ- $\mathrm{SO}_{3}$ quenched 30,40 and $55 \%$ of the SiNP fluorescence, respectively. At 10 quenchers per NP, nearly $80 \%$ of the SiNP fluorescence was quenched for all three quenchers. Thus, all three quenchers displayed capacity to bind SiNPs nonspecifically, though this behaviour depended slightly on the nature of the polar group.

\section{FRET to covalently grafted quenchers}

In a second step, the quenching ability of these amphiphilic BHQs, being covalently attached to the surface of SiNPs, was investigated. In one approach, rhodamine B doped SiNPs bearing an azide group at the terminal end of the Pluronic F127 (Pluronic) were prepared. ${ }^{\mathbf{4 4}}$ The functionalization of the SiNPs with the clickable quenchers by CuAAC "click" chemistry was not efficient, as we could not see significant differences between the samples with and without Cu-catalyst. This could be explained by the poor efficiency of the "click" reaction at low concentrations in the presence of oxygen. Higher efficiency would require the use of a special chelated $\mathrm{Cu}(\mathrm{I})$ catalyst. ${ }^{45} \mathrm{In}$ a second approach, Pluronic- $\mathrm{N}_{3}$ was directly functionalised with the quenchers by CuAAC click chemistry prior to the formulation of SiNPs. The reaction proceeded smoothly and provided pure bi-functionalised Pluronic F127 (Scheme 2) according to NMR. Then, the modified Pluronic polymer was used to prepare SiNPs using the same protocol. Previously, it was estimated that a single SiNP was constituted of $\sim 50$ Pluronic polymers, ${ }^{46,47}$ which corresponded to $\sim 100$ functionalizable sites per particle.

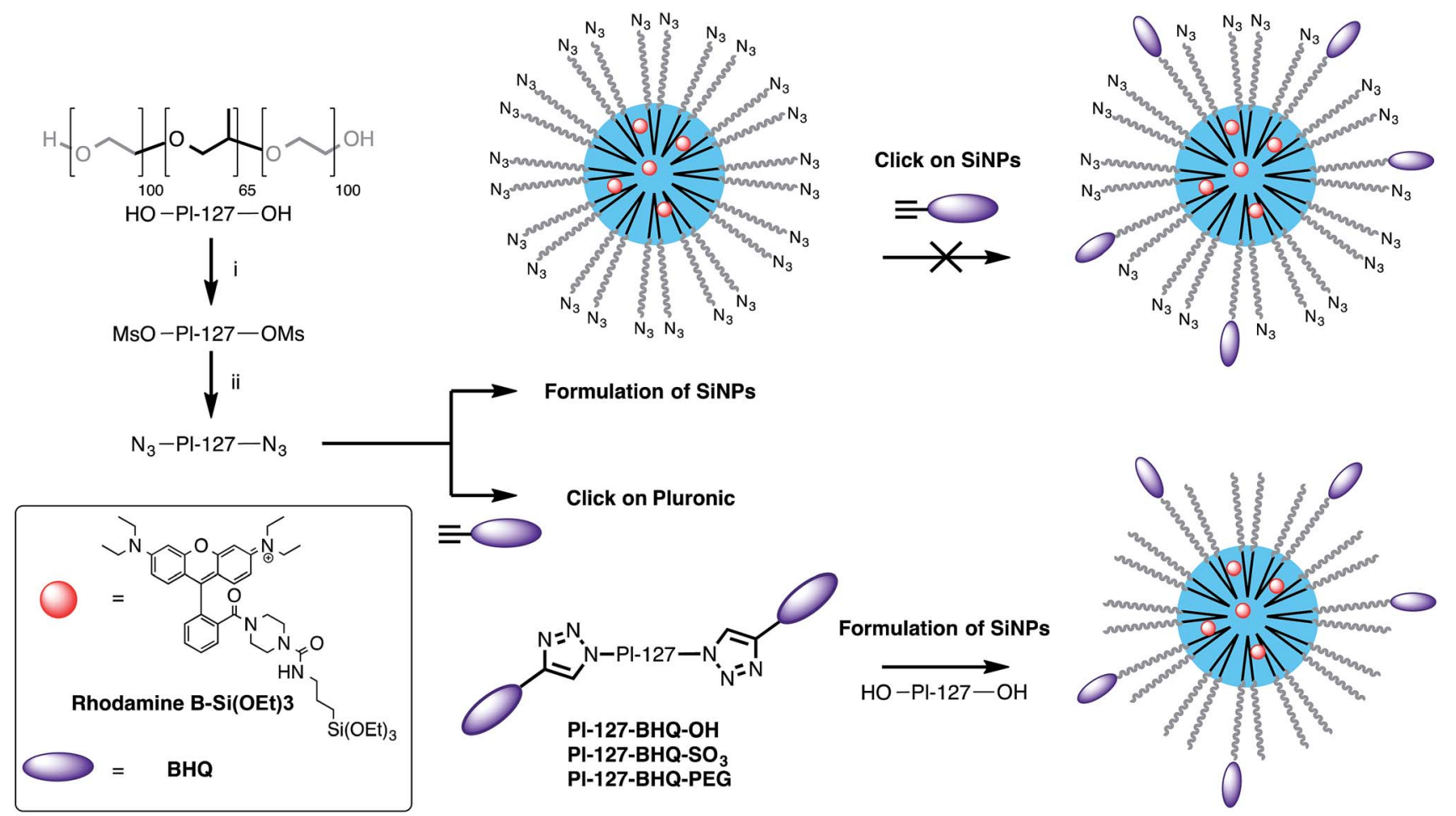

Scheme 2 Two different approaches for the preparation of functionalised SiNPs by CuAAC click chemistry. (i) $\mathrm{MsCl}_{1} \mathrm{NEt}_{3}$, DCM; (ii) $\mathrm{NaN}_{3}$, $\mathrm{CH}_{3} \mathrm{CN}$. 
Therefore, SiNPs bearing 0, 2, 4, 8 and 16 BHQs at their surface were readily obtained by mixing Pluronic and Pluronic-BHQ in appropriate ratios prior to formulation, i.e. $0,2,4,8$ and $16 \%$ of Pluronic-BHQ in total Pluronic, respectively. Dynamic light scattering (DLS) measurements showed that the Pluronic-BHQ molecules did not affect the size $(24 \mathrm{~nm})$ and the low polydispersity value (typically $<0.1$ ) of the obtained SiNPs (Table 1 ). This important result shows that Pluronic modified with

Table 1 Hydrodynamic diameter and polydispersity of the different SiNPs as measured by DLS

\begin{tabular}{llll}
\hline NPs & BHQ (\%) & Diameter (nm) & Polydispersity (PDI) \\
\hline SiNPs & 0 & 22 & 0.02 \\
SiNPs-N $_{3}$ & 0 & 22 & 0.1 \\
SiNPs-BHQ-OH $^{\prime}$ & 16 & 23 & 0.01 \\
SiNPs-SS-BHQ-OH & 16 & 25 & 0.1 \\
SiNPs-SS-BHQ-OH & 32 & 36 & 0.28 \\
SiNPs-BHQ-PEG & 16 & 24 & 0.01 \\
SiNPs-SS-BHQ-PEG & 16 & 24 & 0.03 \\
SiNPs-SS-BHQ-PEG & 32 & 25 & 0.06 \\
SiNPs-BHQ-SO $_{3}$ & 16 & 24 & 0.01 \\
SiNPs-SS-BHQ-SO $_{3}$ & 16 & 29 & 0.25 \\
SiNPs-SS-BHQ-SO $_{3}$ & 32 & 26 & 0.3
\end{tabular}

quencher can be readily used as a building block to prepare functionalized SiNPs with preserved size characteristics.

The increase in the fraction of Pluronic-BHQ during particle synthesis resulted in the gradual increase in the absorbance of the obtained SiNPs as well as broadening of the absorption band with appearance of a shoulder above $600 \mathrm{~nm}$ (Fig. 3A). These spectral changes correspond to the increased contribution of the absorption of the BHQ quenchers, which confirms their successful grafting to SiNPs. Fluorescence measurements of the obtained SiNPs-BHQs showed that an increase in the number of BHQ molecules per particle leads to a gradual decrease of the fluorescence intensity (Fig. 3B and C). Despite their different polar groups, the quenching efficiency was similar for all three quenchers, so that 16 BHQ molecules per particle quenched more than $80 \%$ of SiNPs fluorescence in all cases (Fig. 3C). The average fluorescence lifetime also showed a gradual decrease upon an increase in the number of BHQ quenchers (Fig. 3D), though this effect was slightly stronger for $\mathrm{BHQ}-\mathrm{OH}$. Overall, the lifetime decreased from $\sim 3.7 \mathrm{~ns}$ in the parent SiNPs up to 1.9-2.1 ns in SiNPs bearing 16 quenchers per particle. It should be noted that the decrease in the lifetime is smaller than the decrease in the fluorescence intensity, namely, $<2$-fold and $\sim 5$-fold, respectively. This discrepancy indicates that FRET is probably not the only mechanism of quenching of
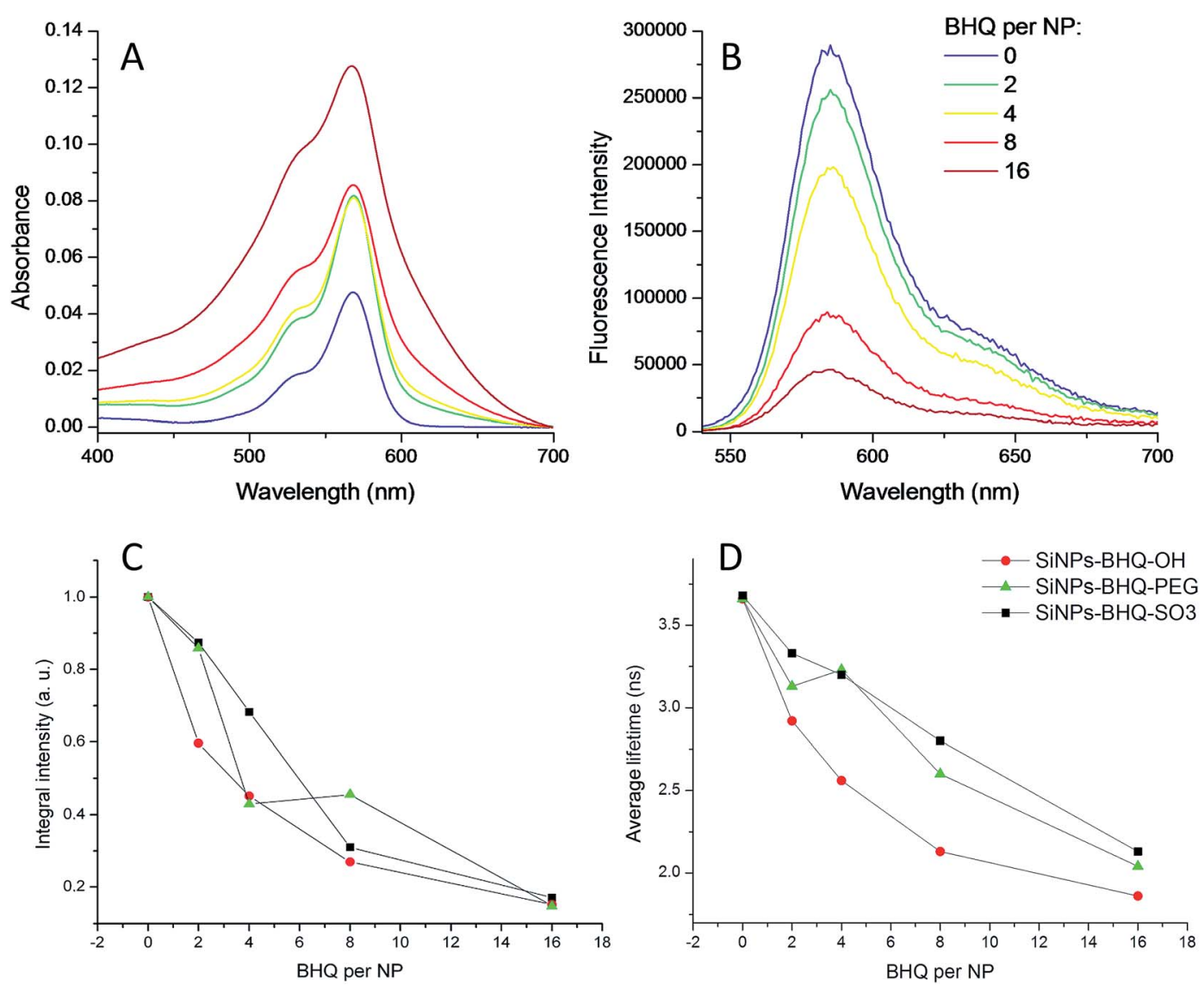

Fig. 3 Spectroscopic properties of SiNPs with grafted $\mathrm{BHQ}$ quenchers. Absorption spectra (A) and emission spectra (B) of $\mathrm{SiNP}-\mathrm{BHQ}-\mathrm{SO} 3(200$ $\mathrm{nM}$ ) with various amounts of $\mathrm{BHQ}$ per particle. Excitation wavelength was $530 \mathrm{~nm}$. (C and D) Quenching effect of the covalently grafted BHQ quenchers on the fluorescence intensity (C) and the average lifetime (D) of SiNPs. 
SiNPs by BHQ. We expect that other mechanisms (electron transfer and excitonic interactions) could produce dark species that decrease the fluorescence intensity without detectable change in the lifetime measurements. Moreover, we cannot exclude a non-homogeneous distribution of dyes and/or quenchers in SiNPs, which could also affect differently fluorescence intensity and the lifetime. Clearly, these experiments show that NPs could be efficiently quenched by a relatively small number of BHQ quenchers and this process can be followed by both intensity and lifetime measurements.

\section{Preparation of nanoprobes and their validation in solution}

Encouraged by the developed synthetic methodology and the quenching efficiency of the new amphiphilic BHQs, we prepared a fluorogenic nanoprobe. The new BHQs quenchers were attached to the terminal PEG chains of the Pluronic F127 through a linker containing a disulfide bond. For this purpose, bromopropanol was converted into alcohol azide 5 , which was activated by carbonyldiimidazole 6 . The latter was coupled to cystamine to give an amino-azide linker 7. Pluronic F127 was converted to a diactive carbamate 8 that was subsequently reacted with 7 to afford the Pluronic azide derivative 9. The latter was clicked to the 3 different amphiphilic BHQs (Scheme 3). The reducible Pluronicquencher conjugates were then mixed with Pluronic F127 in ratios $4 / 21$ and 8/17 prior to the formulation to obtain SiNPs with respectively 16 and 32 BHQs at their surface. These ratios were chosen to insure an efficient quenching and therefore an enhanced turn-ON effect upon reduction.

As expected, the fluorescence intensity decreased many-fold for all three quenchers and the effect was particularly strong with $32 \%$ of quenchers per particle (Fig. S1 $\dagger$ ). To evaluate the ability of these nano-constructs to sense a reductive environment, they were incubated with $20 \mathrm{mM}$ TCEP (pH was adjusted to 7.4 by sodium hydroxide) or $40 \mathrm{mM}$ glutathione and their fluorescence intensity was monitored over one hour. For all quenchers used at $32 \%$, TCEP produced a strong increase in the fluorescence intensity on the time scale of $1 \mathrm{~h}$ (Fig. 4A). The fluorescence increase was lower for SiNPs with lower \% of the quencher $(16 \%)$ and no increase was observed for SiNPs without grafted quencher (Fig. 4B, S2 and S3 $\dagger$ ). Addition of glutathione also produced fluorescence increase when the quencher was grafted to SiNPs, though the fluorescence intensity increased slower, probably because glutathione is a weaker reducing agent than TCEP (Fig. S4 $\dagger$ ). Thus, for SiNPs with $32 \% \mathrm{BHQ}^{-\mathrm{SO}_{3}}$ in presence of $40 \mathrm{mM}$ glutathione, the fluorescence intensity reached a plateau only after $10 \mathrm{~h}$ with a 12-fold enhancement (Fig. S5 $\dagger$ ), which is close to the value obtained in the presence of TCEP in 1 hour (Fig. 4A). The fluorescence intensity of the nanosensors (with $\mathrm{S}-\mathrm{S}$ bond) in the absence of reducing agent was nearly invariant for at least $20 \mathrm{~min}$, which demonstrated their stability in the phosphate buffer medium (Fig. S6 $†$ ). An invariant fluorescence over time was also observed for BHQ-free SiNPs in the presence of glutathione, thus, excluding a direct effect of the reducing agent on SiNPs (Fig. S7†). Finally, in our control SiNPs$\mathrm{BHQ}-\mathrm{OH}$ without reducible linker, no fluorescence increase was observed in the presence of glutathione (Fig. S8†). Taken together, these results suggest that the reducing agents cleave the S-S bonds, which leads to the liberation of the quenchers and thus to the increase in the SiNPs fluorescence.

The three nanosensors (at 32\% of grafting) displayed significantly different turn-ON effect efficiencies upon reduction as their fluorescence enhancement varied from 3- to 13-fold after one hour in the presence of TCEP (Fig. 4A) and 1.5 to 3.5fold after one hour with glutathione (Fig. S4 $\dagger$ ). The following

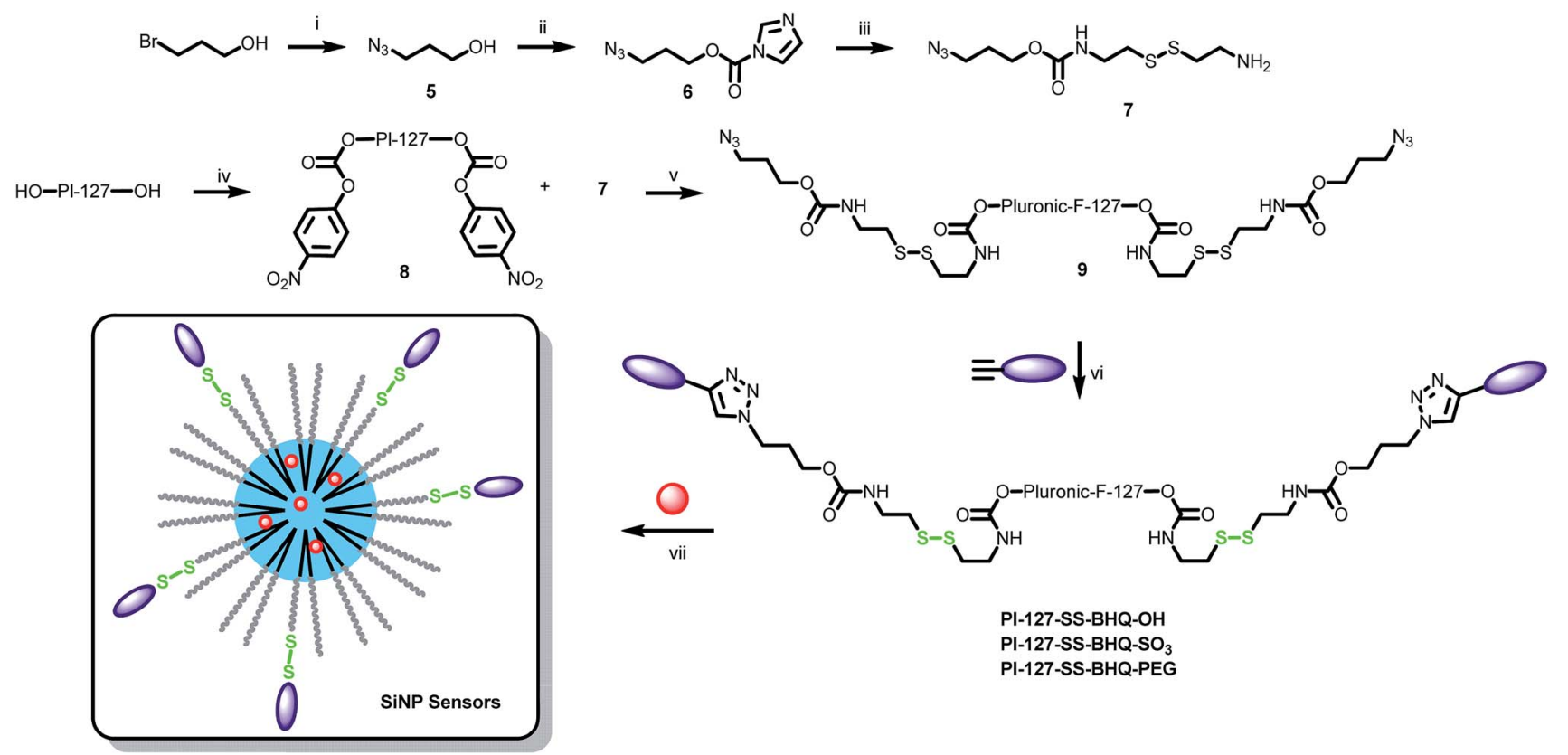

Scheme 3 Synthesis of the reducible SiNPs nanosensors. (i) $\mathrm{NaN}_{3}$, DMF; (ii) carbonyldiimidazole, EtOAc. (iii) Cystamine, DCM; (iv) 4-nitrophenyl chloroformate, pyridine, DCM; (v) DIEA, $\mathrm{CH}_{3} \mathrm{CN}$; (vi) $\mathrm{BHQ}, \mathrm{CuSO}_{4} \cdot 5 \mathrm{H}_{2} \mathrm{O}, \mathrm{AscNa}$, dioxane/water. (vii) Pluronic F127, rhodamine B-Si(OEt) 3 , AcOH, TEOS, TMSCl. 

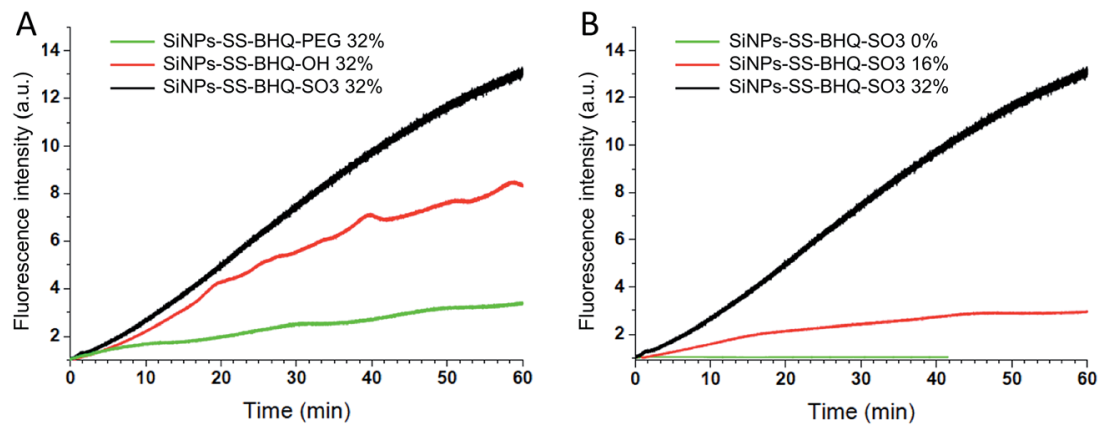

Fig. 4 Effect of TCEP on the fluorescence of the fluorogenic SiNPs. (A) Fluorescence increase as a function of time of $\mathrm{BHQ}-\mathrm{SO}_{3} \mathrm{SiNPs}(20 \mathrm{nM})$ in the presence of $20 \mathrm{mM}$ TCEP in phosphate buffer $20 \mathrm{mM} \mathrm{pH}$ 7.4. (B) Fluorescence increase as a function of time of SiNPs bearing 32\% BHQ-OH (red), $\mathrm{BHQ}-\mathrm{SO}_{3}$ (black) and $\mathrm{BHQ}-\mathrm{PEG}$ (green) $(20 \mathrm{nM})$ in the presence of $20 \mathrm{mM}$ TCEP in phosphate buffer $20 \mathrm{mM}$ pH 7.4. Excitation and emission wavelengths were $530 \mathrm{~nm}$ and $580 \mathrm{~nm}$, respectively.
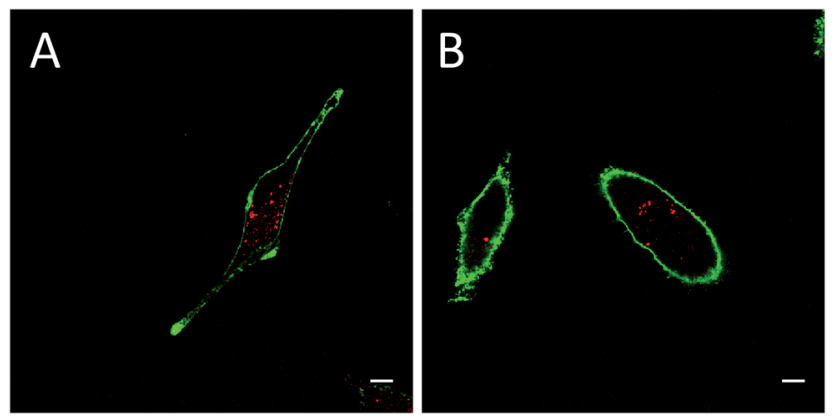

Fig. 5 Confocal laser scanning microscopy images of SiNP-0\% BHQ (A) and $\mathrm{SiNP}-32 \%-\mathrm{SS}-\mathrm{BHQ}-\mathrm{SO}_{3}$ (B) incubated at $50 \mathrm{nM}$ for $2 \mathrm{~h}$ in the presence of HeLa cells. SiNPs were excited at $561 \mathrm{~nm}$ and were detected in the 567-700 $\mathrm{nm}$ range (red color). The plasma membrane was stained by WGA-AlexaFluor®488 (green). Scale bar is $10 \mu \mathrm{m}$.

ranking of $\mathrm{BHQ}$ efficiency in terms of turn-ON effect for the nanosensors could be drawn up: $\mathrm{BHQ}-\mathrm{SO}_{3}>\mathrm{BHQ}-\mathrm{OH}>\mathrm{BHQ}-$ PEG. This tendency can be explained by the fact that, in these systems, an efficient turn-ON effect is ensured by three synergetic factors: (1) the accessibility of the S-S bond; (2) the capacity of BHQ to quench NPs and (3) the capacity of the BHQ to escape from SiNPs after $\mathrm{S}-\mathrm{S}$ bond reduction. For all three quenchers we expect that the access to S-S bond to the reductive agent should be similar. Moreover, all three quenchers at high percentage of grafting showed similar quenching efficiency. Therefore, the observed differences are probably related to the differences in desorption of the quenchers from the SiNPs after $\mathrm{S}-\mathrm{S}$ bond reduction. The presence of a negative charge brought by the sulfate group of $\mathrm{BHQ}-\mathrm{SO}_{3}$ is likely favorable to the dissociation of the BHQ from the fluorescent SiNP, that have a negative zeta potential, leading to an efficient de-quenching of this latter. By contrast, BHQ-PEG and BHQ-OH are noncharged which can explain their lower efficiency compared to $\mathrm{BHQ}-\mathrm{SO}_{3}$ due to less efficient desorption from SiNPs after S-S bond reduction. More unexpected was the significant difference in efficiencies between BHQ-PEG and BHQ-OH. Indeed BHQ$\mathrm{OH}$ was shown to be $>2$ times more efficient than its PEGylated version despite the fact that the latter bears two triazole moieties as well as a $\mathrm{PEG}_{8}$ which enhance its polarity.

\section{Validation in living cells}

The efficient turn-ON effect observed in the SiNP nanoprobes bearing $32 \% \mathrm{BHQ}-\mathrm{SO}_{3}$ led us to investigate their ability to sense the reductive environment in living cells. HeLa cells were incubated for 2 hours in the presence of SiNPs with no quencher and SiNPs with $32 \%$ of $\mathrm{BHQ}-\mathrm{SO}_{3}$. In both cases the cells showed intracellular dotted fluorescence, which can be ambiguously assigned to internalized SiNPs (Fig. 5). The internalization of these nanoprobes probably took place by endocytosis, as suggested by the earlier data using analogous SiNPs. ${ }^{48}$ As SiNPs with $32 \%$ BHQ- $\mathrm{SO}_{3}$ are very poorly fluorescent in the intact form, the observation of fluorescence inside the cells comparable to that of control NPs without grafted quencher indicates that the internalized nanoprobes probably turned ON their fluorescence due to the reductive environment present in cytosol and endosomes of living cells. ${ }^{\mathbf{4 1 , 4 9}}$ However, as intensity response cannot provide definite answer regarding the particle turn-ON, we performed Fluorescence Lifetime Imaging Microscopy (FLIM), which can provide direct information about changes in FRET. ${ }^{\mathbf{5 0}, 51}$

In our FLIM studies, we first compared the images of suspensions of control SiNPs and SiNPs bearing 32\% of BHQ$\mathrm{SO}_{3}$ quenchers (Fig. 6). They showed clearly different pseudocolors (blue and yellow-green), with average lifetimes around 2.9 and $1.8 \mathrm{~ns}$. Thus, in line with our time-resolved studies (see above), the BHQ-bearing SiNPs showed significantly shorter lifetime due to FRET quenching process. Then, we incubated these two types of SiNPs with HeLa cells for $2 \mathrm{~h}$. The obtained FLIM images showed strong intracellular fluorescence in form of dots, indicating good internalization of both types of SiNPs (Fig. 6). However, in the case of SiNPs bearing $\mathrm{BHQ}-\mathrm{SO}_{3}$, the pseudo-color showed clear heterogeneity (Fig. 6D), ranging between long lifetime of control SiNPs (blue) and the short lifetime of SiNPs with $32 \%$ of $\mathrm{BHQ}-\mathrm{SO}_{3}$ (yellow-green). This result suggests that after internalization, some part of the nanoprobes underwent reductive cleavage at the $\mathrm{S}-\mathrm{S}$ bond, which produced de-quenched SiNPs with lifetime close to control SiNPs. Thus, FLIM images validated the response of our nanoprobes to the reductive stimulus in the living cells. Moreover, we showed that FLIM imaging applied to FRET 


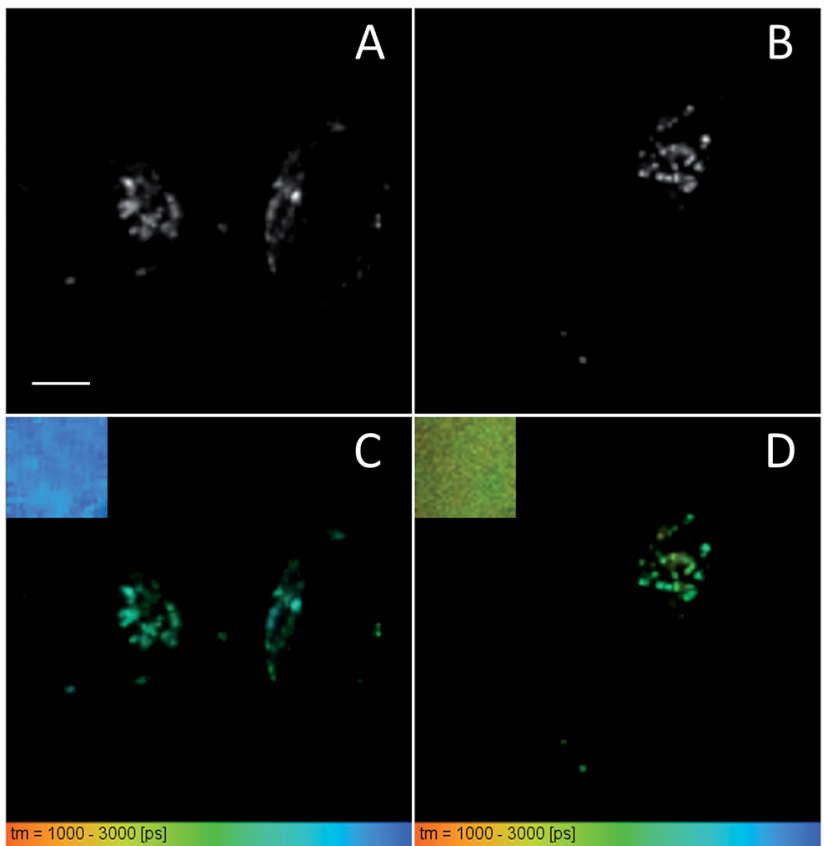

Fig. 6 Intensity (A and $B$ ) and FLIM (C and D) images of SiNP-O\% BHQ $\left(\mathrm{A}\right.$ and $\mathrm{C}$ ) and $\mathrm{SiNP}-32 \%-\mathrm{SS}-\mathrm{BHQ}-\mathrm{SO}_{3}(\mathrm{~B}$ and $\mathrm{D})$ incubated at $50 \mathrm{nM}$ for $2 \mathrm{~h}$ in the presence of HeLa cells. Insets of (C) and (D) at the top left corner show the FLIM image of corresponding SiNPs without (SiNP-0\% $\mathrm{BHQ}$ ) and with $\mathrm{BHQ}$ quencher (SiNP-32\%-SS-BHQ-SO 3 ) in solution. Scale bar is $10 \mu \mathrm{m}$.

nanoprobes is complementary to intensity measurements, because FLIM provides information about FRET efficiency, thus describing the probe response to the reductive environment.

\section{Materials and methods}

Milli-Q water (Millipore) was used in all experiments. All starting materials for synthesis were purchased from Alfa Aesar, Sigma Aldrich or TCI Europe and used as received unless stated otherwise. Synthesis of the BHQ and the Pluronic F127 derivatives are described in the ESI. $\dagger$ NMR spectra were recorded on a Bruker Avance III $400 \mathrm{MHz}$ spectrometer. Mass spectra were obtained using an Agilent Q-TOF 6520 mass spectrometer. DLS measurements were performed on a Zetasizer Nano series DTS 1060 (Malvern Instruments S.A.). Absorption and emission spectra were recorded on a Cary 400 Scan ultraviolet-visible spectrophotometer (Varian) and a FluoroMax-3 spectrofluorometer (Horiba Jobin Yvon) equipped with a thermostated cell compartment, respectively. For standard recording of fluorescence spectra, the excitation wavelength was set to $530 \mathrm{~nm}$ and emission was recorded from 540 to $700 \mathrm{~nm}$. The solutions of TCEP and glutathione were prepared in $20 \mathrm{mM}$ phosphate buffer solutions (pH 7.4) and the $\mathrm{pH}$ was readjusted to 7.4.

\section{Cell culture}

HeLa cells (ATCC CCL-2) were grown in DMEM (GibcoInvitrogen), supplemented with $10 \%$ fetal bovine serum (Lonza) and $1 \%$ antibiotic solution (penicillin-streptomycin, Gibco-
Invitrogen) at $37{ }^{\circ} \mathrm{C}$ in humidified atmosphere containing $5 \%$ $\mathrm{CO}_{2}$. Cells were seeded onto a chambered coverglass (IBiDi) at a density of $5 \times 10^{4}$ cells per well $24 \mathrm{~h}$ before the microscopy measurements. For imaging, the culture medium was removed and the attached cells were washed with Opti-MEM (GibcoInvitrogen). Next, a freshly prepared solution of NPs (50 nM) in Opti-MEM was added to the cells, which were incubated for 2 or $24 \mathrm{~h}$. The medium was then removed and washed 2 times with HBSS and replaced by HBSS for imaging. Cell plasma membrane staining with wheat-germ agglutinin-Alexa488 (50 nM) was done at room temperature $10 \mathrm{~min}$ before the measurements.

\section{Fluorescence lifetime measurements in solution}

Time-resolved fluorescence measurements were performed with the time-correlated, single-photon counting technique using the excitation pulses at $480 \mathrm{~nm}$ provided by a pulsepicked frequency doubled Ti-sapphire laser (Tsunami, Spectra Physics) pumped by a Millenia X laser (Spectra Physics). The emission was collected through a polarizer set at the magic angle and an $8 \mathrm{~nm}$ band-pass monochromator (Jobin-Yvon H10) at $582 \mathrm{~nm}$. The single-photon events were detected with a microchannel plate photomultiplier (Hamamatsu) coupled to a pulse pre-amplifier HFAC (Becker-Hickl $\mathrm{GmbH}$ ) and recorded on a time correlated single photon counting board SPC-130 (Becker-Hickl $\mathrm{GmbH}$ ). The instrumental response function (IRF) was recorded using a polished aluminum reflector, and its full-width at half-maximum was $\sim 40$ ps. Experimentally measured fluorescence decays were deconvoluted with the instrumental response function and fitted to retrieve the most probable lifetime distribution using the maximum entropy method (Pulse 5 software). ${ }^{52,53}$ In all cases, the $\chi^{2}$ values were close to 1 , indicating an optimal fit.

\section{Fluorescence lifetime imaging microscopy}

Fluorescence microscopy experiments were performed on a home-built two-photon laser scanning setup based on an Olympus IX70 inverted microscope with an Olympus $60 \times 1.2$ NA water immersion objective. ${ }^{54}$ Two-photon excitation was provided by a Ti-sapphire (Tsunami, Spectra Physics) or an Insight DeepSee (Spectra Physics) laser. Imaging was carried out using two fast galvo-mirrors operating in the descanned fluorescence collection mode. Photons were detected with an avalanche photodiode (APD SPCM-AQR-14-FC, PerkinElmer) which was connected to a time-correlated single photon counting (TCSPC) module (SPC830, Becker and Hickl) operating in reversed start-stop mode. The typical acquisition time was $60 \mathrm{~s}$ with an excitation power around $5 \mathrm{~mW}(830 \mathrm{~nm})$ at the laser output. Subsequent data analysis using the software SPC Image (Becker and Hickl) allowed us to extract the fluorescence lifetimes from the decays, and their visualization in a FLIM image, using an arbitrary color scale.

\section{Conclusions}

Preparation of FRET-based sensors using nanoparticles remains a challenge because particle size is generally larger than the 
Förster distance. The problem can be solved by using very small NPs and a large number of quencher moieties. Here, we designed a nanosensor where fluorescent dye-doped silica NPs (SiNP) bear covalently linked quenchers through a disulfide bond. We prepared $\sim 20 \mathrm{~nm}$ silica NPs doped with rhodamine B dyes and grafted with BHQ-2 type quenchers. It was found that optimal quenching ( $>10$-fold) requires 16 to 32 quenchers at the SiNP surface. Moreover, the amphiphilic nature of the quencher was found to play a key role in the design of these nanosensors. Timeresolved fluorescence measurements suggested in addition to the expected FRET mechanism, the presence of dark species due to static quenching. In the presence of TCEP or glutathione, SiNPs strongly increase their fluorescence due to the reduction of the disulfide bonds and separation of the quenchers from the particle. Laser scanning confocal imaging showed that particles can be internalized in cells. Fluorescence lifetime measurements (FLIM) revealed populations of internalized NPs with short and long lifetimes corresponding to weakly and strongly reduced SiNPs, respectively. Our data confirmed that the SiNPs nanoprobes lose their quenchers in response to the intracellular reductive environment. This work provides insights into design of FRET-based nanoparticle probes and demonstrates suitability of FLIM for characterization of these nanoprobes in living cells.

\section{Acknowledgements}

This work was supported by the European Research Council ERC Consolidator grant BrightSens 648528, Université de Strasbourg (IdEX 2015, W15RAT68), Global Grant Spinner 2013, Emilia-Romagna region and by Marco Polo grant, University of Bologna.

\section{References}

1 B. N. G. Giepmans, S. R. Adams, M. H. Ellisman and R. Y. Tsien, Science, 2006, 312, 217-224.

2 X. Li, X. Gao, W. Shi and H. Ma, Chem. Rev., 2014, 114, 590659.

3 L. E. Santos-Figueroa, M. E. Moragues, E. Climent, A. Agostini, R. Martínez-Máñez and F. Sancenón, Chem. Soc. Rev., 2013, 42, 3489-3613.

4 L. D. Lavis and R. T. Raines, ACS Chem. Biol., 2008, 3, 142155.

5 O. S. Wolfbeis, Chem. Soc. Rev., 2015, 44, 4743-4768.

6 P. D. Howes, R. Chandrawati and M. M. Stevens, Science, 2014, 346, 1247390.

7 A. Reisch and A. S. Klymchenko, Small, 2016, 12, 1968-1992. 8 W. C. W. Chan and S. M. Nie, Science, 1998, 281, 2016-2018. 9 X. Michalet, F. F. Pinaud, L. A. Bentolila, J. M. Tsay, S. Doose, J. J. Li, G. Sundaresan, A. M. Wu, S. S. Gambhir and S. Weiss, Science, 2005, 307, 538-544.

10 M. Montalti, L. Prodi, E. Rampazzo and N. Zaccheroni, Chem. Soc. Rev., 2014, 43, 4243-4268.

11 S. Bonacchi, D. Genovese, R. Juris, M. Montalti, L. Prodi, E. Rampazzo and N. Zaccheroni, Angew. Chem., Int. Ed., 2011, 50, 4056-4066.
12 J. E. Lee, N. Lee, T. Kim, J. Kim and T. Hyeon, Acc. Chem. Res., 2011, 44, 893-902.

13 K. M. Wang, X. X. He, X. H. Yang and H. Shi, Acc. Chem. Res., 2013, 46, 1367-1376.

14 V. N. Mochalin, O. Shenderova, D. Ho and Y. Gogotsi, Nat. Nanotechnol., 2012, 7, 11-23.

15 Y. Rong, C. Wu, J. Yu, X. Zhang, F. Ye, M. Zeigler, M. E. Gallina, I.-C. Wu, Y. Zhang, Y.-H. Chan, W. Sun, K. Uvdal and D. T. Chiu, ACS Nano, 2013, 7, 376-384.

16 C. Wu and D. T. Chiu, Angew. Chem., Int. Ed., 2013, 52, 30863109.

17 K. Li and B. Liu, Chem. Soc. Rev., 2014, 43, 6570-6597.

18 A. Reisch, P. Didier, L. Richert, S. Oncul, Y. Arntz, Y. Mely and A. S. Klymchenko, Nat. Commun., 2014, 5, 4089.

19 K. Trofymchuk, A. Reisch, I. Shulov, Y. Mely and A. S. Klymchenko, Nanoscale, 2014, 6, 12934-12942.

20 D. Genovese, E. Rampazzo, S. Bonacchi, M. Montalti, N. Zaccheroni and L. Prodi, Nanoscale, 2014, 6, 3022-3036.

21 K. E. Sapsford, L. Berti and I. L. Medintz, Angew. Chem., Int. Ed., 2006, 45, 4562-4588.

22 J. L. Fan, M. M. Hu, P. Zhan and X. J. Peng, Chem. Soc. Rev., 2013, 42, 29-43.

23 A. E. Albers, V. S. Okreglak and C. J. Chang, J. Am. Chem. Soc., 2006, 128, 9640-9641.

24 G. Leriche, G. Budin, Z. Darwich, D. Weltin, Y. Mely, A. S. Klymchenko and A. Wagner, Chem. Commun., 2012, 48, 3224-3226.

25 I. L. Medintz, A. R. Clapp, H. Mattoussi, E. R. Goldman, B. Fisher and J. M. Mauro, Nat. Mater., 2003, 2, 630-638.

26 A. I. Zamaleeva, M. Collot, E. Bahembera, C. Tisseyre, P. Rostaing, A. V. Yakovlev, M. Oheim, M. de Waard, J.-M. Mallet and A. Feltz, Nano Lett., 2014, 14, 2994-3001.

27 J. Valanciunaite, A. S. Klymchenko, A. Skripka, L. Richert, S. Steponkiene, G. Streckyte, Y. Mely and R. Rotomskis, RSC Adv., 2014, 4, 52270-52278.

28 K. Trofymchuk, L. Prodi, A. Reisch, Y. Mély, K. Altenhöner, J. Mattay and A. S. Klymchenko, J. Phys. Chem. Lett., 2015, 6, 2259-2264.

29 M. Arca, C. Caltagirone, G. De Filippo, M. Formica, V. Fusi, L. Giorgi, V. Lippolis, L. Prodi, E. Rampazzo, M. A. Scorciapino, M. Sgarzi and N. Zaccheroni, Chem. Commun., 2014, 50, 15259-15262.

30 E. Oliveira, D. Genovese, R. Juris, N. Zaccheroni, J. L. Capelo, M. M. M. Raposo, S. P. G. Costa, L. Prodi and C. Lodeiro, Inorg. Chem., 2011, 50, 8834-8849.

31 S. Kim, H. E. Pudavar and P. N. Prasad, Chem. Commun., 2006, 2071-2073.

32 L. S. Khabbaz, M. Hassanzadeh-Khayyat, P. Zaree, M. Ramezani, K. Abnous and S. M. Taghdisi, Anal. Methods, 2015, 7, 8611-8616.

33 S. M. Taghdisi, N. M. Danesh, H. R. Beheshti, M. Ramezani and K. Abnous, Nanoscale, 2016, 8, 3439-3446.

34 F. Gao, P. Cui, X. X. Chen, Q. Q. Ye, M. G. Li and L. Wang, Analyst, 2011, 136, 3973-3980.

35 D. Genovese, M. Montalti, L. Prodi, E. Rampazzo, N. Zaccheroni, O. Tosic, K. Altenhoner, F. May and J. Mattay, Chem. Commun., 2011, 47, 10975-10977. 
36 E. Rampazzo, S. Bonacchi, R. Juris, M. Montalti, D. Genovese, N. Zaccheroni, L. Prodi, D. C. Rambaldi, A. Zattoni and P. Reschiglian, J. Phys. Chem. B, 2010, 114, 14605-14613.

37 X. X. He, Y. S. Wang, K. M. Wang, M. Chen and S. Y. Chen, Anal. Chem., 2012, 84, 9056-9064.

38 J. Wang, P. P. Gao, X. X. Yang, T. T. Wang and C. Z. Huang, J. Mater. Chem. B, 2014, 2, 4379-4386.

39 X. X. He, L. Hai, J. Su, K. M. Wang and X. Wu, Nanoscale, 2011, 3, 2936-2942.

40 Y. X. Tang, H. Hu, M. G. Zhang, J. B. Song, L. M. Nie, S. J. Wang, G. Niu, P. Huang, G. M. Lu and X. Y. Chen, Nanoscale, 2015, 7, 6304-6310.

41 J.-H. Ryu, R. T. Chacko, S. Jiwpanich, S. Bickerton, R. P. Babu and S. Thayumanavan, J. Am. Chem. Soc., 2010, 132, 1722717235.

42 G. Gasparini, E.-K. Bang, G. Molinard, D. V. Tulumello, S. Ward, S. O. Kelley, A. Roux, N. Sakai and S. Matile, J. Am. Chem. Soc., 2014, 136, 6069-6074.

43 Y. Niko, Y. Arntz, Y. Mely, G.-i. Konishi and A. S. Klymchenko, Chem.-Eur. J., 2014, 20, 16473-16477.
44 L. Bromberg and M. Temchenko, Langmuir, 1999, 15, 86338639.

45 Q. Wang, T. R. Chan, R. Hilgraf, V. V. Fokin, K. B. Sharpless and M. G. Finn, J. Am. Chem. Soc., 2003, 125, 3192-3193.

46 P. K. Sharma and S. R. Bhatia, Int. J. Pharm., 2004, 278, 361377.

47 G. Wanka, H. Hoffmann and W. Ulbricht, Macromolecules, 1994, 27, 4145-4159.

48 E. Rampazzo, R. Voltan, L. Petrizza, N. Zaccheroni, L. Prodi, F. Casciano, G. Zauli and P. Secchiero, Nanoscale, 2013, 5, 7897-7905.

49 J. Yang, H. Chen, I. R. Vlahov, J.-X. Cheng and P. S. Low, Proc. Natl. Acad. Sci. U. S. A., 2006, 103, 13872-13877.

$50 \mathrm{H}$. Wallrabe and A. Periasamy, Curr. Opin. Biotechnol., 2005, 16, 19-27.

51 K. Suhling, P. M. W. French and D. Phillips, Photochem. Photobiol. Sci., 2005, 4, 13-22.

52 A. K. Livesey and J. C. Brochon, Biophys. J., 1987, 52, 693-706. 53 J. C. Brochon, Methods Enzymol., 1994, 240, 262-311.

54 J. P. Clamme, J. Azoulay and Y. Mely, Biophys. J., 2003, 84, 1960-1968. 